\title{
0 retalho músculo-cutâneo peitoral maior na reconstrução do câncer de cabeça e pescoço
}

\section{The pectoralis major musculocutaneous flap in head and neck cancer reconstruction}

Elio Gilberto Pfuetzenreiter Júnior', Cláudio Rogério Alves de Andrade², Carlos Neutzling Lehn', Rogério Aparecido Dedivitis ${ }^{1}$

\section{Resumo}

Introdução: Descrito inicialmente por Ariyan na década de 1970, o retalho de peitoral maior tem grande aceitação nas reconstruções por câncer em cabeça e pescoço pela sua versatilidade. Ele é irrigado pela artéria toracoacromial com uma circulação adicional proveniente da artéria torácica lateral. Método e casuística: 0 retalho foi utilizado para a reconstrução de 104 pacientes com câncer de cabeça e pescoço, sendo: reconstrução de boca/língua (57); reconstrução do trânsito faringo-esofágico (12); reparo de perda de pele cervical (15); reconstrução de faringe (10); reparo de perda de pele de face (7); e reparo de fístula faringo-cutânea (3). Resultados: Necrose total do retalho foi observada em quatro pacientes, necrose parcial e deiscência em 22 pacientes e epidermólise em 31. Conclusão: 0 retalho foi funcionalmente adequado para a reconstrução de grandes defeitos cirúrgicos.

Palavras-chave: Retalhos cirúrgicos; procedimentos cirúrgicos reconstrutivos; neoplasias de cabeça e pescoço; complicações pós-operatórias.

\section{Abstract}

Introduction: The pectoralis major flap was initially described by Ariyan in the 1970s. It has high acceptance due to its versatility for head and neck cancer reconstruction. It is supplied by the thoracoacromial artery with additional circulation provided by the lateral thoracic artery. Methods and subjects: We performed that flap for the reconstruction of 104 patients with head and neck cancer, as following: mouth/tongue reconstruction (57); pharyngoesophageal transit reconstruction (12); repair of cervical skin loss (15); pharynx reconstruction (10); repair of facial skin loss (7); and repair of pharyngocutaneous fistula (3). Results: Total necrosis of the flap was observed in four patients, partial necrosis with dehiscence in 22 and epidermolysis in 31. Conclusions: The flap was functionally adequate for the repair of large surgical defects.

Keywords: Surgical flaps; reconstructive surgical procedures; head and neck neoplasms; postoperative complications.

Recebido: 16/01/2008

Revisado: $10 / 08 / 2008$

Aprovado: $11 / 08 / 2008$

\footnotetext{
Programa de Pós-Graduação em Ciências da Saúde do Hospital Heliópolis (HOSPHEL), São Paulo (SP)

2 Departamento de Cirurgia de Cabeça e Pescoço e Otorrinolaringologia do HOSPHEL

Trabalho realizado no Programa de Pós-Graduação em Ciências da Saúde, do HOSPHEL

Endereço para correspondência: Rogério Aparecido Dedivitis, Rua Cônego Xavier, 276, CEP 04231-030 - São Paulo (SP), Fax: (13) $3223-5550$ / 3221 -

1514,E-mail: dedivitis.hns@uol.com.br
} 


\section{Introdução}

O tratamento do câncer em cabeça e pescoço muitas vezes requer ressecções extensas e retalhos para sua reconstrução. Uma vez que proporciona bons resultados cosméticos, a reconstrução imediata é preferível. O retalho músculo-cutâneo de peitoral maior (RMPM) é versátil e de ampla utilização na região da cabeça e pescoço, desde os trabalhos de Ariyan $^{1,2}$. O músculo peitoral maior é irrigado principalmente pela artéria toracoacromial.

Em uma série que avaliou 96 casos, Wadwongtham et al. ${ }^{3}$ descrevram complicações maiores do RMPM em 17,7\% dos pacientes, incluindo perda total do retalho, perda parcial de pele, fístula orocutânea, deiscência e exposição de placa. O índice global de complicações foi de $54,2 \%$, sendo a maioria abordada de forma conservadora. Em um período de 20 anos, Milenovic et al. ${ }^{4}$ utilizaram 506 RMPM em 500 pacientes para reconstrução intra-oral (387 casos - 77\%) e faríngea (78 casos 15\%). Defeitos ósseos ocorreram em 65 pacientes. Em 31 (6\%) o RMPM foi usado em combinação com outros retalhos (deltopeitoral, de língua, trapézio e microvascularizados). Complicações ocorreram em 168 retalhos (33\%), mas necrose total foi vista somente em 10 (2\%). Tratamento cirúrgico das complicações foi indicado em 87 casos (17\%). Em outro estudo de 27 pacientes com reconstrução de câncer de cavidade oral com placa de reconstrução de titânio e RMPM, Salvatori et al. ${ }^{5}$ não encontraram óbito perioperatório e relataram seis casos de exposição da placa, sendo precoce em quatro casos e tardia em dois. Dois casos foram corrigidos cirurgicamente, com um índice final de sucesso de $85 \%$. A maioria dos pacientes considerou o resultado estético aceitável. Em estudo com 25 pacientes, as indicações para tal retalho por El-Marakby ${ }^{6}$ foram: cavidade oral (dez pacientes); orofaringe e hipofaringe (cinco pacientes) e pescoço ou face (dez pacientes). De 26 procedimentos, 22 foram reconstruções primárias e quatro foram "resgates". Deiscência, infecção, hematoma, seroma, perda parcial ou total, fistula e complicações no sítio doador foram verificados em 15 pacientes (60\%), associando-se a indicações para "resgate", comorbidades e indicações para cavidade oral. Segundo Chaturvedi et al. ${ }^{7}$, em defeitos que requerem ampla reconstrução

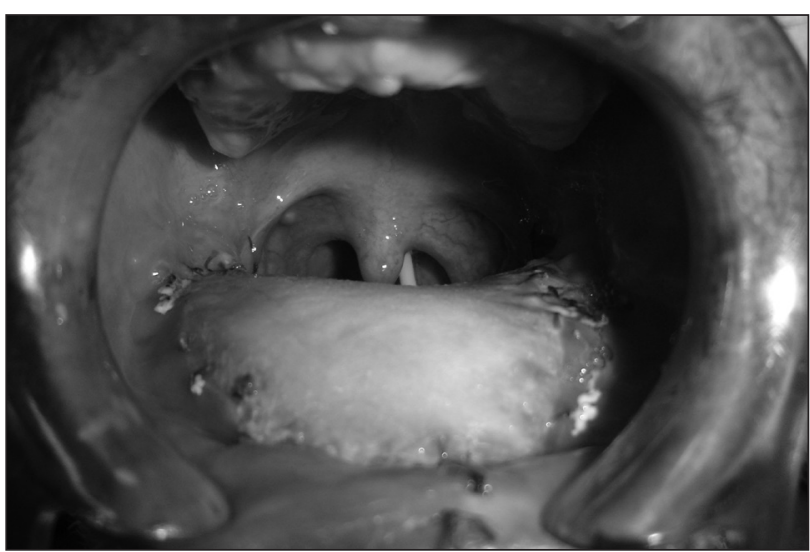

Figura 1 - 0 retalho músculo-cutâneo na reconstrução do soalho da boca de pele e mucosa, o RMPM pode ser usado em duas camadas ou ainda em combinação com o retalho deltopeitoral, especialmente quando não há condições de utilizar-se retalho microvascularizado.

Nos últimos anos, o retalho livre antebraquial tem substituído o RMPM na reconstrução de partes moles de boca e orofaringe. Em estudo pareando dois grupos de pacientes, o menor custo da internação hospitalar foi comparável ao maior custo do procedimento cirúrgico do retalho antebraquial microvascularizado. Assim, com custos finais comparáveis e melhor resultado funcional, o retalho antebraquial é considerado como primeira opção reconstrutiva ${ }^{8}$. No entanto, quando as condições regionais ou gerais do paciente não permitem a utilização de retalho microvascularizado, inclusive nas reconstruções de "resgate" após deiscência ou falha prévia, retalhos pediculados devem ser considerados ${ }^{9}$. Apesar do uso amplo dos retalhos microvascularizados, o RMPM ainda é considerada a principal opção reconstrutiva para câncer de cabeça e pescoço em muitos centros.

O objetivo desse estudo foi apresentar as complicações locais do uso do retalho músculo-cutâneo de peitoral maior, em grandes reconstruções de cabeça e pescoço em um serviço de referência terciária, bem como apresentar a abordagem realizada frente às complicações encontradas.

\section{Casuística e métodos}

Foi realizada reconstrução com RMPM em 104 pacientes cirurgicamente tratados entre janeiro de 1988 e dezembro de 2006, no Serviço de Cirurgia de Cabeça e Pescoço e Otorrinolaringologia do Hospital Heliópolis (SP), sendo 97 homens, com idade variando entre 32 e 77 anos (mediana de 61). As indicações foram: reconstrução de boca/ língua (57) (Figura 1); reconstrução do trânsito faringo-esofágico (12); reparo de perda de pele cervical (15); reconstrução de faringe (10); reparo de perda de pele de face (7) (Figura 2); e reparo de fístula faringocutânea (3). Sessenta e dois pacientes foram submetidos à radioterapia pós-operatória e dez foram submetidas à radioterapia pré-operatória, com doses maiores do que 5.000 cGy. O sítio doador foi fechado primariamente em todos os casos.

Foram avaliadas retrospectivamente as seguintes complicações: deiscência (parcial ou total); necrose (epidermólise, parcial ou total); fístula para a pele; e deiscência do sítio doador. Verificou-se a conduta frente às complicações encontradas (conservadora ou cirúrgica) e os resultados dessa conduta.

Esta pesquisa foi aprovada pelo Comitê de Ética em Pesquisa da Irmandade da Santa Casa da Misericórdia de Santos, com parecer 49/2008.

\section{Resultados}

A complicação mais comum observada após a reconstrução com RMPM foi perda parcial ou necrose da pele do retalho com deiscência parcial da linha de sutura no reparo do soalho da boca (epidermólise) 31 pacientes. Vinte e dois pacientes desenvolveram deiscência parcial e 
fístula. Houve quatro casos de necrose total na reconstrução do soalho da boca e trânsito faringo-esofágico. Funcionalmente, nos outros pacientes, o retalho foi adequado para a reconstrução e para o reparo da maioria dos defeitos cirúrgicos.

Re-sutura foi realizada em oito casos; retalho deltopeitoral foi realizada em sete pacientes, sendo associada à faringostomia em cinco. Todos esses 15 pacientes submetidos ao segundo procedimento reconstrutivo apresentaram resultado final satisfatório. Seroma de sítio doador foi clinicamente observado em um paciente.

\section{Discussão}

O RMPM é versátil e a reconstrução de grandes defeitos na cabeça e pescoço pode ser facilmente realizada utilizando um procedimento em tempo único. A utilização de um retalho com músculo não-irradiado em um sítio não-irradiado facilita bons resultados.

O retalho encontra bom suporte no segmento muscular na reconstrução de defeitos intra-orais, mesmo na vigência de perda de pele e deiscência parcial. Nesses casos de epidermólise, os pacientes costumam evoluir satisfatoriamente com terapia local, formando-se tecido de granulação na área. Apesar da irrigação subcutânea por ramos arteriais, a saliva contribui para perda da camada superficial da pele. Por outro lado, é possível atingir bons resultados ao utilizar-se um retalho fásciomuscular, ou seja, sem o uso da pele do tórax, para o reparo de defeitos intra-orais. Uma vantagem adicional da não rotação da pele do tórax junto com o retalho é evitar o crescimento de pelos dentro da boca. Entretanto, como boa parte desses pacientes vai submeter-se à radioterapia adjuvante, tal modalidade terapêutica acaba por causar epilação.

A incidência de complicações varia de 16 a $41 \%{ }^{10}$. Em um universo de 104 pacientes, encontramos 3,8\% de perda total por necrose. No total, a rotação de um segundo retalho foi necessária em $6,7 \%$ dos casos e re-sutura do RMPM foi feita em 7,6\%. Devido às dimensões amplas do retalho muitas vezes necessárias, o achado de necroses parciais e epidermólise é comum e resolve-se de forma conservadora, sem adicionar tempo de internação ou morbidade significativa aos pacientes. Apesar da crescente utilização de retalhos livres microvasculares, o RMPM continua a ser um retalho muito confiável e ainda amplamente utilizado.

Complicações no sítio doador, como deiscência mínima, hematoma, seroma e infecção, têm sido infreqüentemente observadas ${ }^{11}$. Em nossa casuística, houve um único caso de seroma, resolvido de forma conservadora.

Encontramos quatro casos de necrose em nossa casuística. Uma das explicações para a necrose do retalho é a compressão extrínseca do

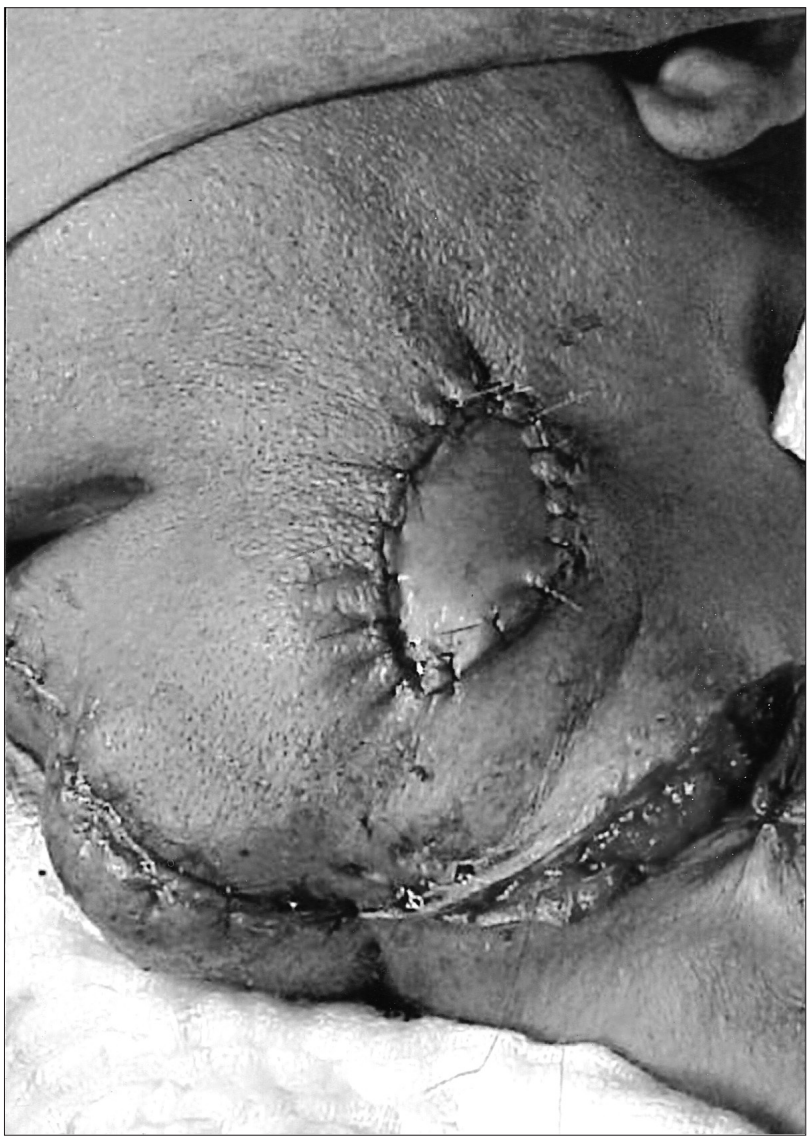

Figura 2 - 0 retalho músculo-cutâneo colocado para reconstrução de pele da face

pedículo vascular, principalmente no túnel subcutâneo por onde o mesmo passa, no nível da clavícula.

Apesar do uso progressivamente mais popular dos retalhos livres microvascularizados, o RMPM segue sendo uma opção bastante confiável e de fácil execução pelo especialista. $O$ retalho antebraquial radial provavelmente seja preferível no reparo de áreas que requeiram opções mais delicadas, como na reconstrução de defeitos de espessura total da região jugal. Por outro lado, é obviamente difícil trabalhar com um retalho espesso de pacientes obesos e de pacientes femininas com mamas volumosas. Tais dificuldades podem ser superadas com o emprego do retalho fásciomuscular, cuja espessura é menor. No caso de pacientes do gênero feminino, os retalhos microvascularizados evitam resultados estéticos ruins na região das mamas por conta da incisão inframamária do RMPM.

Em conclusão, o retalho ficou funcionalmente adequado tanto para a reconstrução da neofaringe quanto no reparo de grandes defeitos cirúrgicos. 


\section{Referências}

1. Ariyan S. The pectoralis major myocutaneous flap. A versatile flap for reconstruction in the head and neck. Plast Reconstr Surg 1979;63(1): 73-81.

2. Ariyan $\mathrm{S}$, Cuono $\mathrm{CB}$. The use of the pectoralis major myocutaneous flap for reconstruction of the large cervical, facial, or cranial defects. Am J Surg 1980;140:503.

3. Wadwongtham W, Isipradit $\mathrm{P}$, Supanakorn S. The pectoralis major myocutaneous flap: applications and complications in head and neck reconstruction. J Med Assoc Thai 2004;87(Suppl 2):S95-9.

4. Milenovic A, Virag M, Uglesic V, Aljinovic-Ratkovic N. The pectoralis major flap in head and neck reconstruction: first 500 patients. J Craniomaxillofac Surg 2006;34(6):340-3.

5. Salvatori P, Motto E, Paradisi S, Zani A, Podrecca S, Molinari R. Oromandibular reconstruction using titanium plate and pectoralis major myocutaneous flap. Acta Otorhinolaryngol Ita 2007;27(5):227-32.

6. El-Marakby HH. The reliability of pectoralis major myocutaneous flap in head and neck reconstruction. J Egypt Natl Canc Inst 2006;18(1):41-50.
7. Chaturvedi P, Pai PS, Pathak KA, Chaukar DA, Deshpande MS, D'Cruz AK. Simultaneous reconstruction of large skin and mucosal defect following head and neck surgery with a single skin paddle pectoralis major myocutaneous flap. J Laryngol Otol 2005;119(4): 303-5.

8. de Bree R, Reith R, Quak JJ, Uyl-de Groot CA, van Agthoven M, Leemans $\mathrm{CR}$. Free radial forearm flap versus pectoralis major myocutaneous flap reconstruction of oral and oropharyngeal defects: a cost analysis. Clin Otolaryngol 2007;32(4):275-82.

9. Okazaki M, Asato H, Takushima A, Sarukawa S, Nakatsuka T, Yamada A et al. Analysis of salvage treatments following the failure of free flap transfer caused by vascular thrombosis in reconstruction for head and neck cancer. Plast Reconstr Surg 2007;119(4):1223-32.

10. Lee YT. Carotid artery protection by pectoralis major muscle flap. Am J Surg 1980;140(3):464.

11. Dedivitis RA, Guimarães AV. Pectoralis major musculocutaneous flap in head and neck cancer reconstruction. World J Surg 2002;26(1): 67-71. 